\title{
Surface-Area-Dependent Electron Transfer Between Isoenergetic 2D Quantum Wells and a Molecular Acceptor
}

\author{
Benjamin T. Diroll, ${ }^{\mathrm{a}}$ Igor Fedin, ${ }^{\mathrm{b}}$ Pierre Darancet, ${ }^{\mathrm{a}}$ Dmitri V. Talapin, ${ }^{\mathrm{a}, \mathrm{b}}$ Richard D. Schaller, ${ }^{\mathrm{a}, c^{*}} \ldots$ \\ ${ }^{a}$ Center for Nanoscale Materials, Argonne National Laboratory, Lemont, IL 60439, United States \\ bDepartment of Chemistry, University of Chicago, Chicago, IL 60637, United States \\ 'Department of Chemistry, Northwestern University, Evanston, IL 60208, United States
}

\section{Materials}

Methylviologen dichloride hydrate (98\%), Cadmium nitrate hydrate (99.9\%), sodium myristate (98.0\%), cadmium acetate dihydrate (98\%), cadmium acetylacetonate (99.9\%), octadecene (90\%), oleic acid (90\%) were purchased from Sigma-Aldrich. All solvents used were ACS grade or higher.

\section{Methods}

Synthesis of Nanoplatelets (NPLs). NPLs were synthesized following literature protocols. ${ }^{1,2}$ Briefly, 170 mg cadmium myristate (synthesized by precipitation in methanol from cadmium nitrate tetrahydrate and sodium myristate $\left.\mathrm{e}^{3}\right), 15 \mathrm{~mL}$ octadecene, and $12 \mathrm{mg}$ Se powder were loaded into a $50 \mathrm{~mL} 3$ neck flask and held under vacuum ( $\sim 1$ torr) at room temperature for 30 minutes, then heated under nitrogen to $240^{\circ} \mathrm{C}$. At $190^{\circ} \mathrm{C}, 40 \mathrm{mg}$ of finely-ground cadmium acetate was rapidly added to the reaction flask under nitrogen -counterflow. Once the reaction temperature reached $240{ }^{\circ} \mathrm{C}$, it was held at that temperature for 10 minutes. To obtain smaller plates, aliquots were collected as the reaction temperature reached $215^{\circ} \mathrm{C}$ and after 2 minutes at $240^{\circ} \mathrm{C}$. Purification was performed by adding $2 \mathrm{~mL}$ oleic acid and $10 \mathrm{~mL}$ hexanes followed by centrifugation at $12000 \mathrm{rpm}$ for 5 minutes. The yellow pellet was redispersed in hexanes. To dissolve NPLs in chloroform, solutions were evaporated to dryness under nitrogen flow and then redissolved in chloroform.

Synthesis of Quantum Dots (QDs). Spherical zinc-blende QDs used in this work were synthesized following a modified literature procedure. ${ }^{3} 31 \mathrm{mg}$ cadmium acetylacetonate, $4 \mathrm{mg}$ Se powder, $5 \mathrm{~mL}$ ODE, and $0.13 \mathrm{~mL}$ oleic acid were loaded into a $25 \mathrm{~mL}$ 3-neck flask and heated to $120^{\circ} \mathrm{C}$ under vacuum for 1 hour. Then, the reaction pot was heated to $190^{\circ} \mathrm{C}$ under nitrogen atmosphere and the heat source was removed upon reaching that temperature, cooling back to room temperature. QDs were precipitated from solution using isopropanol and centrifugation (repeated with hexanes/isopropanol), and dispersed into hexanes. The first excitonic absorption maximum of the sample is at $511 \mathrm{~nm}$.

Preparation of Samples for Quenching Experiments. Methylviologen dichloride salt was dissolved in a mixture of 2:1 chloroform:methanol at a concentration of 4 millimolar and subsequently diluted with clean solvent (of the same mixture) for dilution studies. $150 \mu \mathrm{L}$ of NPLs or QDs dissolved in chloroform with an optical density of approximately 0.4 at $400 \mathrm{~nm}$ were mixed with $1 \mu \mathrm{L}$ of $2: 1$ chloroform:methanol solution either blank (for control measurements) or with dissolved methylviologen dichloride. 
Steady-State Optical Data. Absorption spectra were collected using a Cary 50 spectrometer. Steadystate emission spectroscopy was performed using a $405 \mathrm{~nm}$ diode (PicoQuant) with emission fibercoupled into a spectrometer equipped with a CCD array.

Time-Resolved Photoluminescence. Time-resolved photoluminescence data was collected using a streak camera with excitation at $2 \mathrm{kHz}$ using the frequency-doubled output (400 nm) of a $35 \mathrm{fs} \mathrm{Ti}$ : sapphire laser.

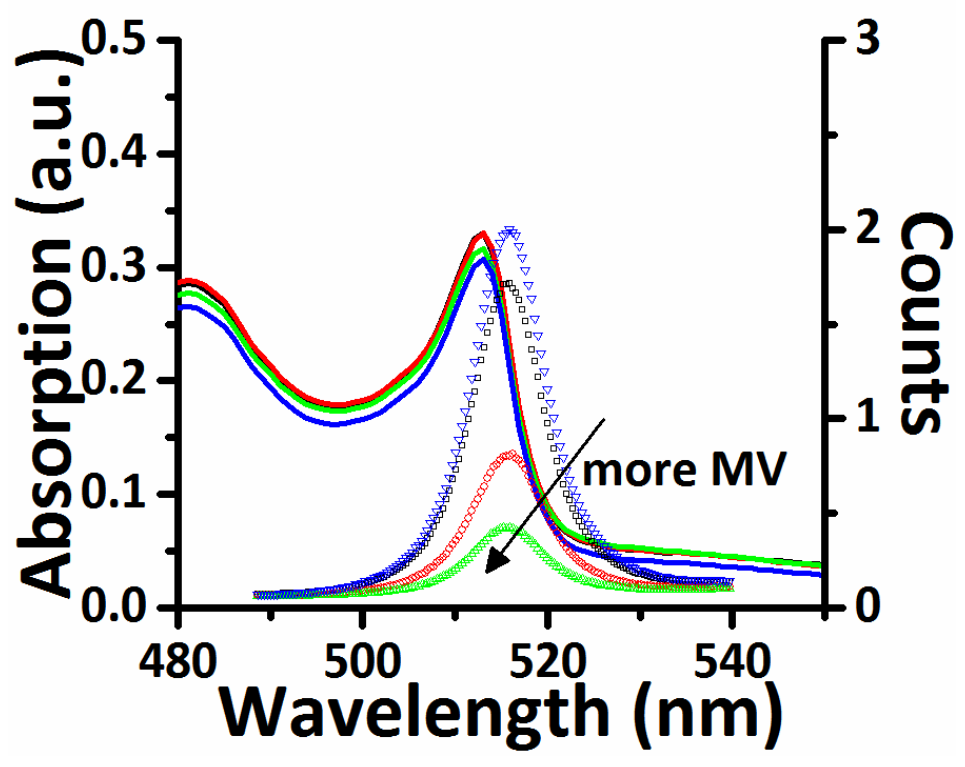

Figure S1. Steady-state absorption (solid lines, left) and steady-state photoluminescence spectra (open symbols, right) of the "large" CdSe nanoplatelet sample upon the addition of methylviologen. Colors indicate the same sample. The arrow indicates the direction of MV addition with respect to the photoluminescence spectra. 


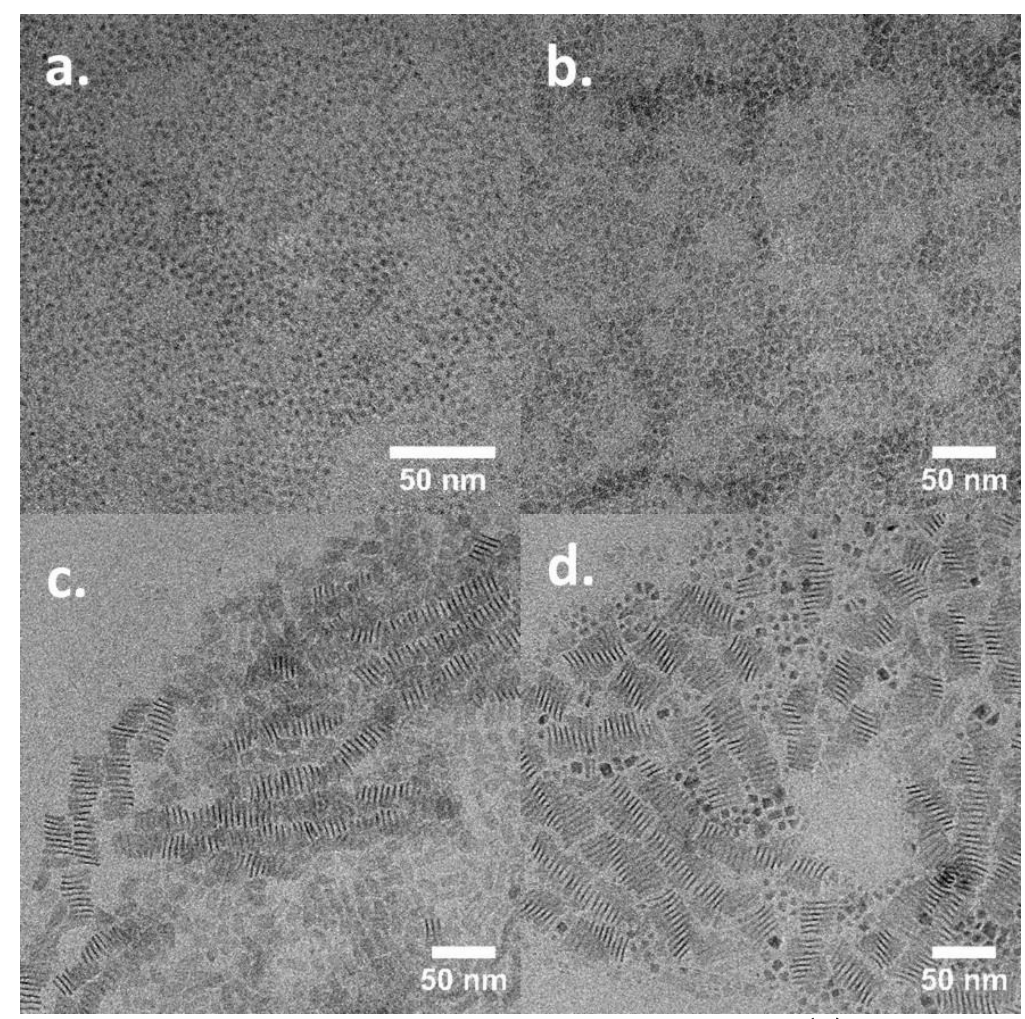

Figure S2. Expanded view of transmission electron micrographs of (a) quantum dots, (b) small, (c) medium, and (d) large nanoplatelets used for spectroscopic studies. Cube impurities in (d) do not contribute to the measurement because they have a distinct band gap from the platelets and therefore a different emission energy. (See also emission spectra in Figure S1.)

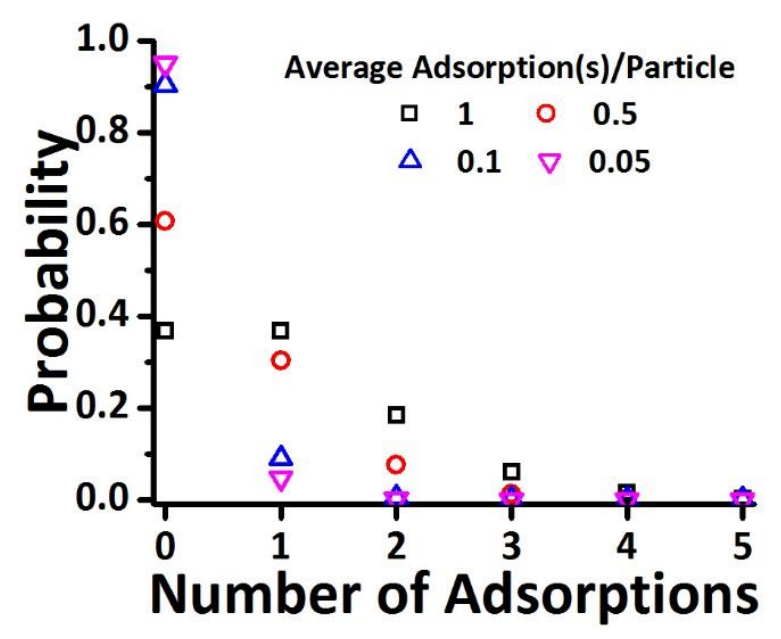

Figure S3. Poisson distribution of adsorption events for given average adsorptions/particle. 


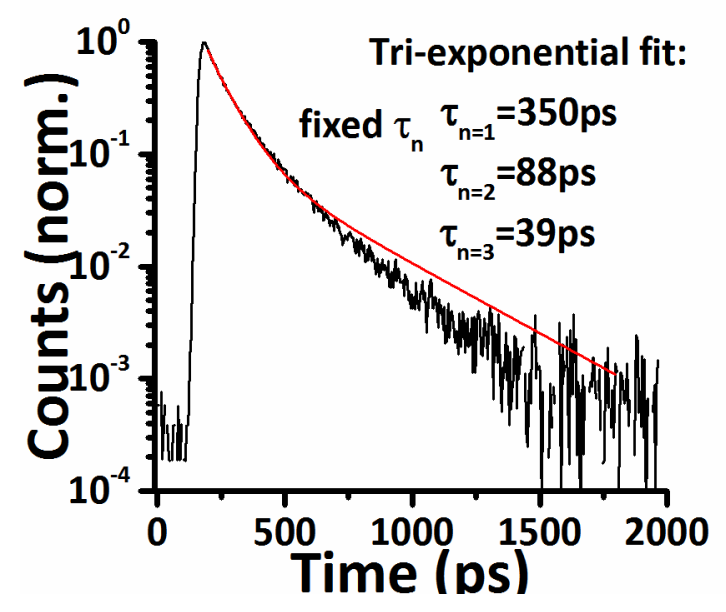

Figure S4. Triexponential fit of MV quenching data of large NPLs collected with an MV concentration of $31 \mu \mathrm{M}$. The red fit line represents the a tri-exponential decay to the data (black) with the three lifetime parameters fixed at the specified values according to $\tau_{n}=\tau_{n=1} / n^{2}$.

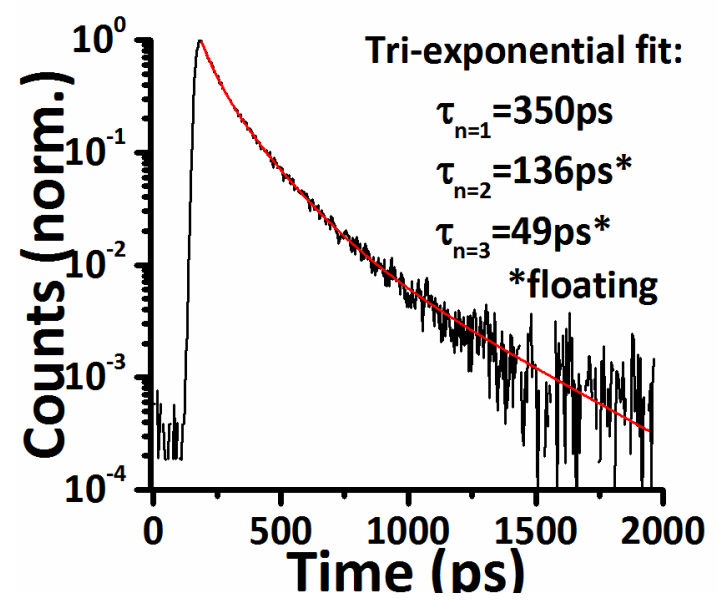

Figure S5. Triexponential fit of MV quenching data of large NPLs collected with an MV concentration of $31 \mu \mathrm{M}$. The red fit line represents the best fit of a tri-exponential decay to the data (black) with only the bimolecular quenching rate fixed. 


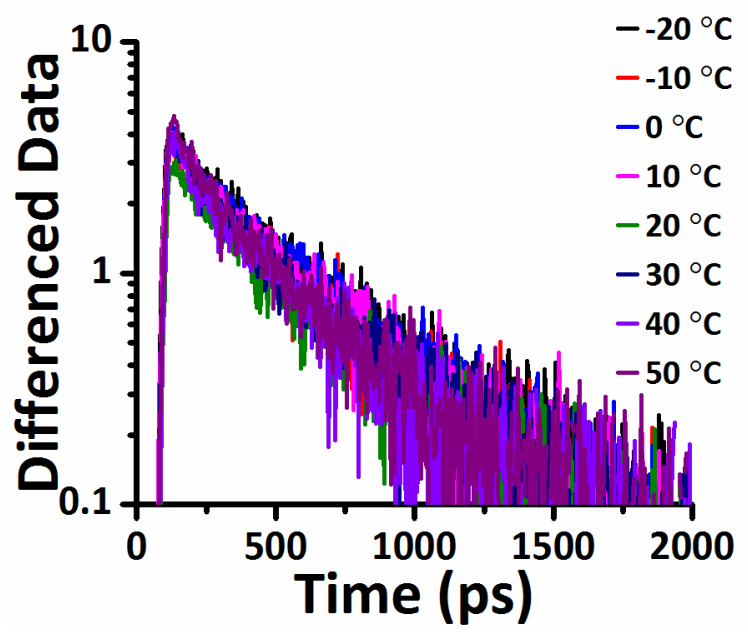

Figure 6. Temperature-dependent differenced data, showing bimolecular CT, for large NPLs.

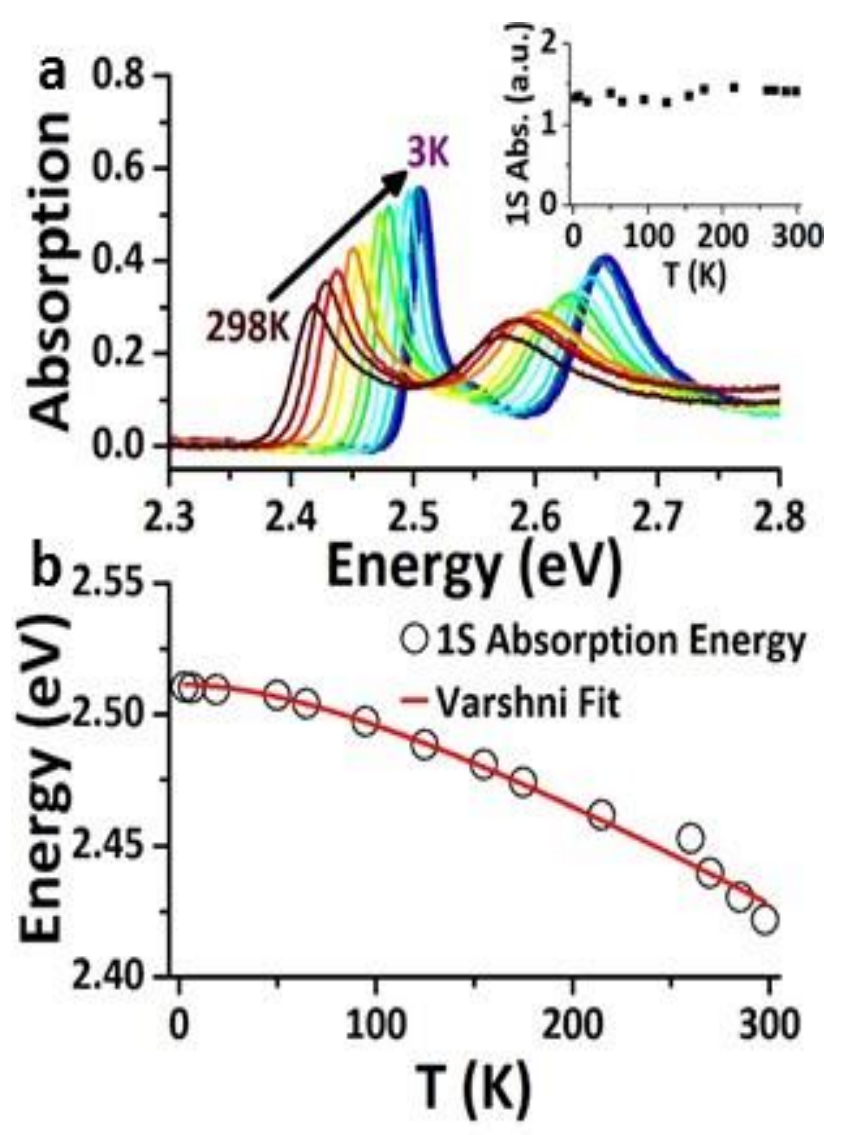

Figure S7. (a) Temperature-dependent absorption spectra collected on a NPL film. Inset shows the integrated absorption of the first excitonic absorption versus temperature determined by Gaussian fitting. (b) Peak energy of the first excitonic absorption versus temperature (open circles) with a Varshni fit (solid line). 


\section{References}

(1) Ithurria, S.; Dubertret, B. J. Am. Chem. Soc. 2008, 130, 16504.

(2) She, C.; Fedin, I.; Dolzhnikov, D. S.; Dahlberg, P. D.; Engel, G. S.; Schaller, R. D.; Talapin, D. V. ACS Nano 2015, 9, 9475.

(3) Yang, Y. A.; Wu, H.; Williams, K. R.; Cao, Y. C. Angew. Chem., Int. Ed. Engl. 2005, 44, 6712. 